\title{
OXIDATIVE METABOLISM AND PHYSICAL PROPERTIES OF THE BLOOD IN ATHLETES
}

\author{
ANDREW K MARTUSEVICH ${ }^{*}$, KONSTANTIN A KARUZIN
}

${ }^{1}$ Laboratory of Medical Biophysics, Privolzhsky Research Medical University, Nizhny Novgorod, Russia. ${ }^{2}$ Bioniq Health-Tech Solutions Ltd., London, United Kingdom. Email: cryst-mart@yandex.ru

Received: 18 May 2020, Revised and Accepted: 30 June 2020

ABSTRACT

Objectives: Monitoring of metabolic status in athletes includes a wide range of different parameters, but not all of them have been fully studied. Thus, the presence of oxidative stress is noted in athletes, but its features are revealed only in fragments. The crystallogenic properties of blood in athletes have not been previously studied.

Methods: The study enrolled a total of 262 athletes (19-29 years old) that are highly qualified in cyclic sports. Furthermore, screening data of apparently healthy non-exercising volunteers of the same age $(n=35)$ were used for control. Blood serum of the athletes and non-exercising volunteers was analyzed for 8-isoprostane level, oxidized low-density lipoproteins, activity of superoxide dismutase, and glutathione peroxidase and reductase. We also studied the level $\alpha$ - and $\beta$-carotenes, $\alpha$ - and $\gamma$-tocopherols, lycopene, lutein, and zeaxanthin. Crystallogenic properties of blood serum were tested with own method.

Results: The complex study demonstrated the presence of shifts in oxidative metabolism and blood serum physical and chemical properties induced by professional sports and manifested both in the status of blood pro- and antioxidative systems and in shifts of biofluids crystallogenic activity. At the same time, the obtained data confirmed the development of oxidative stress in qualified athletes.

Conclusion: Our data shown that there are some shifts of oxidative metabolism and crystallogenic properties of blood plasma in professional athletes.

Keywords: Athletes, Metabolism, Free radical processes, Plasma crystallization, Biocrystallomics.

(C) 2020 The Authors. Published by Innovare Academic Sciences Pvt Ltd. This is an open access article under the CC BY license (http://creativecommons. org/licenses/by/4. 0/) DOI: http://dx.doi.org/10.22159/ajpcr.2020.v13i9.38572

\section{INTRODUCTION}

High-performance sport is one of the national priorities whose successful implementation requires the establishment of athlete training and support systems [1-5]. A medical screening system is mainly focused on the identification and management of conditions, while substantially less attention is paid to the study of the capacity of the athlete's body to adapt to extreme loads [1-5]. Conventionally, the adaptation includes all the types of congenital and acquired adaptive activities implemented at cellular, systemic, and organismic levels $[6,7]$. Physiological adaptation is a process of maintaining the functional status of organs and systems, including maintaining performance efficiency in extreme conditions $[6,8]$. One of the key factors of this process is the continuous regeneration of cellular structures which is directly dependent on the behavior of free-radical processes [8]. Free-radical processes undergo substantial changes under various physiological and pathological conditions $[6,9]$. Oxidative metabolism of the whole body and its functional constituents is maintained by an aggregated complex of prooxidant and antioxidant systems comprising enzymatic and non-enzymatic (bioradical and non-radical) components [8-11]. Ubiquity of oxidative metabolism predetermines its involvement in the pathogenesis of the absolute majority of diseases, and on the other hand, it provides extensive opportunities for correction [11-13]. Maladaptation of oxidative metabolism is termed as oxidative stress which is considered as a special syndrome $[9,11,14-16]$.

The aim of the study was to investigate the status of oxidative metabolism as well as the blood physical and chemical properties of athletes in comparison with non-trained people.

\section{METHODS}

The study was performed on 262 highly qualified athletes, which specializing in cyclic sports (competitive skiing, rowing, bicycling, track and field athletics, and orienteering) and hold sports titles. The studied athletes were aged 19-29 years. Furthermore, screening data of apparently healthy non-exercising volunteers of the same age $(n=35)$ were used for control. The study was approved by a local Ethics Committee of the Burnazyan Federal Medical Biophysical Center (No. 18 dated October 12, 2015). All the studied patients had signed informed consent to participate in the study.

Blood serum of the athletes and non-exercising volunteers was analyzed for 8-isoprostane levels using 8-isoprostane enzymelinked immunosorbent assay (ELISA) test kit (USBiological, USA). An assay of oxidized low-density lipoproteins (oLDL) was performed by competitive ELISA method in a microplate using the Evolis automated immunoassay analyzer (Bio-Rad, Germany - USA) with Biomedica Gruppe reagents. Superoxide dismutase (SOD) activity in the blood plasma was assessed by inhibition of adrenaline autooxidation in a carbonate buffer $(\mathrm{pH}=10.0)$ while adding samples of blood hemolysate (1:50) following the method of M. Sun, S. Zigman (1978). The study of blood level of $\alpha$ - and $\beta$-carotene as well as $\alpha$ - and $\gamma$-tocopherol was performed by a standard method using analyzers as per the method of EA, Moisenok et al. (2009). The estimation of the concentration of tissue-specific antioxidants (lycopene, lutein, and zeaxanthin) in blood samples was performed by chromatography-mass spectrometry using a technique described by Grigoriev (2005) and Batsukova and Yaremko (2015).

All the samples were centrifuged for 15 min (2500 g; Multifuge 1S-R centrifuge) to obtain blood serum. Then, $100 \mu \mathrm{l}$ of blood serum was applied on a microscope slide, and slides with a dried biological fluid were prepared following the crystalloscopic method allowing to evaluate crystallogenic activity of the biological material $[17,18]$. The dried slides were evaluated morphologically (by describing special features of the structure of the biological fluid) and by visual parameters 
(using our own system of parameters) [17]. The basic visual parameters rated by a scale were crystallizability (reflecting a quantitative aspect of crystallization, i.e., density of crystal elements in facias), structure index (defines complexity of structure formation), facia destruction degree (as an indicator of a qualitative aspect of the process, i.e., structure formation regularity), and clarity of facia marginal zone. The evaluation covered three concurrently obtained facias with calculation of these parameter values. The whole facia areas were analyzed. The sample images were obtained using Levenhuk kit.

Data acquired in the course of the study are processed using Statistica 6.1 software package for Windows. Arithmetic average (X), error of average $(\mathrm{m})$, and average absolute deviation $(\sigma)$ were calculated for each sampling. Shapiro-Wilk test was used to test data for normal distribution. A method of one-way analysis of variance was used to compare sampling units of several experimental samples with a control one. The differences were considered significant at $\mathrm{p}<0.05$.

\section{RESULTS AND DISCUSSION}

It was found that the majority of the studied metabolic parameters are significantly different in qualified athletes as compared with those characteristic for non-exercising volunteers. Thus, people with regular exercising showed a significantly higher level of 8-isoprostane in the blood plasma (Fig. 1); and the value of this parameter was in 1.25 times higher as compared to the control group $(\mathrm{p}<0.05)$. It is known that 8 -isoprostane plasma concentration is now considered as an integral laboratory marker of oxidative stress $[9,16]$. That is why the identified trend is indicative of excessive stimulation of free-radical oxidation processes induced by intensive physical exercise.

At the same time, the level of oLDLs in professional athletes was on $16 \%$ lower than in non-exercising volunteers ( $<<0.05 ;$ Fig. 2 ), which seems to be due to a primary effect of the studied factor not on lipid peroxidation but on oxidative damage of other macromolecules, particularly, on oxidative protein modification. This is indirectly proven by the nature of the SOD (one of the major antioxidant enzymes) activity shifts observed in highly trained athletes. Representatives of this group showed moderate inhibition of this enzyme's catalytic capacities as compared to the level found in the control group (by $8 \% ; \mathrm{p}=0.072$ ).

The above parameter level shifts indicate an active involvement of the enzyme in scavenging free radicals (by removing superoxide anion radicals from the biological fluid) on the one hand and can reflect partial modification of SOD as a large protein molecule.

Furthermore, changes in plasma concentrations of non-tissue-specific non-enzymatic antioxidants also indicate pronounced activation of freeradical processes in qualified athletes. In particular, $\alpha$ - and $\gamma$-tocopherol levels in individuals regularly having intensive physical exercise are significantly lower as compared to non-exercising individuals (Fig. 3).

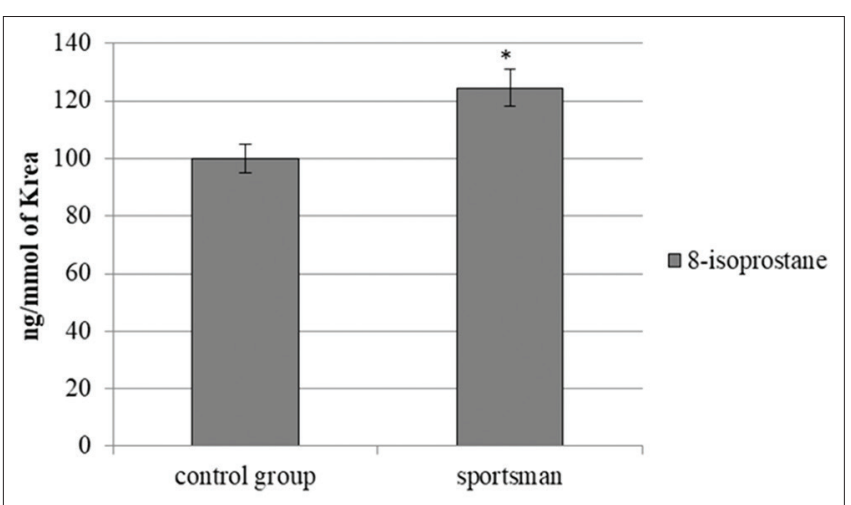

Fig. 1: Level of 8-isoprostane in blood serum of athletes and non-trained people (“*” - differences between control group and athletes are statistically valuable, $p<0.05$ )
The most significant trend is observed in terms of $\gamma$-tocopherol, which concentration decreases 1.68 fold in athletes as compared to the representatives of the control group, while $\alpha$-tocopherol is 1.23 -fold lower ( $\mathrm{p}<0.05$ for both parameters).

It is noteworthy that we observed not only absolute decrease in levels of both tocopherols but also given the lipophilic nature of tocopherols, a decrease in the ratio of Vitamin E level/concentration of plasma cholesterol, which is 1.25 -fold lower in trained individuals as compared to the representatives of the control group $(\mathrm{p}<0.05)$.

Similar but less pronounced changes were registered for the other group of non-selective non-enzymatic antioxidants, that is, carotenes. It was found that $\alpha$-carotene concentrations decrease more significantly than $\beta$-carotene concentrations in plasma of professional athletes (1.3-fold and 1.1-fold, respectively; $\mathrm{p}<0.05$ for both parameters). This further confirms the deficiency of antioxidant potential which develops under continuous intensive physical exercise and the onset of oxidative stress under such conditions.

Said trend fully applies to tissue-specific antioxidants. In particular, plasma levels of zeaxanthin, lycopene, and lutein were significantly decreased in qualified athletes as compared to non-exercising volunteers (up to 1.9-fold; $\mathrm{p}<0.05$ ).

Concurrently with the evaluation of parameters representing oxidative metabolism status, we studied athlete and non-exercising individual blood serum samples for crystallogenic activity integrally reflecting physical and chemical properties of biological fluid and its composition. We found

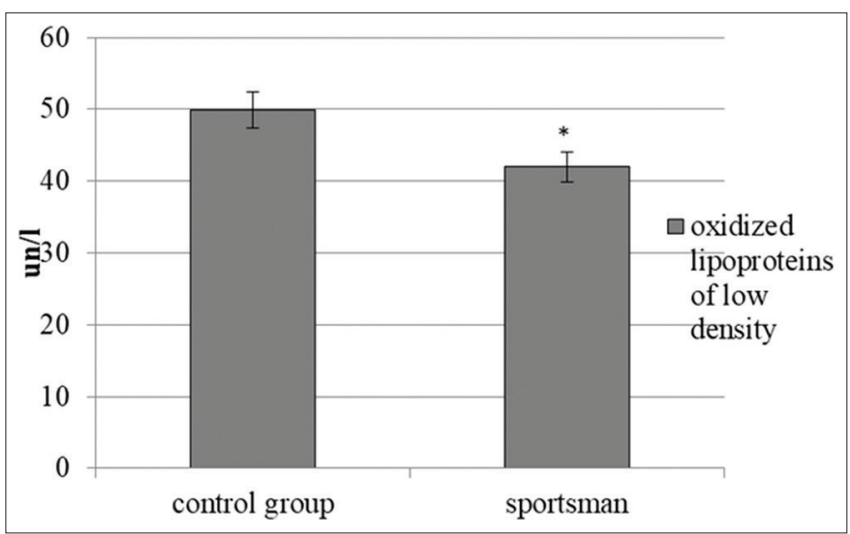

Fig. 2: Level of oxidized lipoproteins of low density in blood serum of athletes and non-trained people ( “*” - differences between control group and athletes are statistically valuable, $\mathbf{p}<0.05$ )

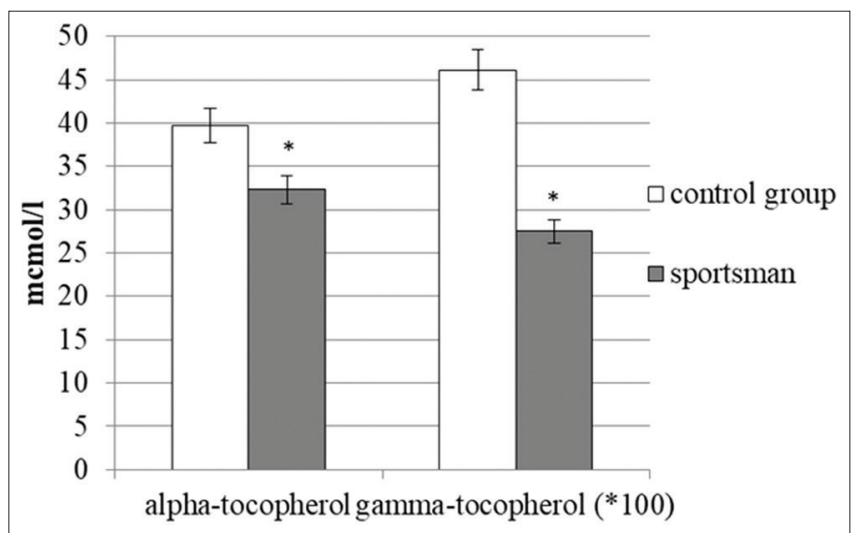

Fig. 3: Level of alpha- and gamma-tocopherols in blood serum of athletes and non-trained people (“*” - differences between control group and athletes are statistically valuable, $p<0.05$ ) 
shifts in all the major visual parameters of blood serum facias of athletes as compared to those of the individuals having no regular physical exercise.

Thus, in comparison with non-exercising volunteers, we observed an apparent trend of increase $(1.22$-fold; $\mathrm{p}<0.05)$ in crystallizability being a quantitative characteristic of the result of biofluid dehydration structuring and demonstrating its activity. This may be due to significant athletes' plasma concentrations of under oxidized products of free-radical processes which stimulation is evidenced by the above data. Such compounds in the biological fluid can take on the role of crystallization centers, and the presence of such compounds promotes the formation of organo-mineral aggregations described as crystal elements at visual evaluation.

The proposed hypothesis is also confirmed by visual parameter changes representing the qualitative aspect of crystallogenesis, that is, structure degree index, allowing to take into consideration the complexity of element structure formation in a dehydrated sample of the blood serum.

The professional athletes also demonstrated higher values of this parameter as compared to the non-exercising volunteers (1.3-fold; $\mathrm{p}<0.05)$. These changes indicate that metabolic adaptation in the course of prolonged intensive physical exercise leads not only to the quantitative stimulation of biological fluid crystallogenic activity but also to complexification of the formed structures. This indicates significant shifts in physical and chemical properties of a biosubstrate.

Evaluation of the facia destruction degree is particularly interesting. Facias destruction is the major criterion of crystallogenesis regularity, now considered indicative of the extent of maladaptivity of changes in biological fluid crystallogenic properties. It was found that dried samples of non-exercising volunteer blood serum showed only moderate signs of facias element destruction, while samples of most qualified athletes showed subtotal destruction of histological slide crystal structures. This was reflected by the degree of facias destruction of $0.64 \pm 0.14$ and $2.12 \pm 0.36$ points in non-exercising and trained individuals, respectively $(p<0.05)$, thus implying the pathological nature of crystallogenesis shifts in athletes, which are induced by regular intensive physical exercise (metabolic maladaptation).

We observed an inverse trend with regards to clarity of marginal zone of serum histological slide. The level of this parameter was lower in qualified athletes as facias margin was narrowed in this group of studied individuals. Based on the known molecular mechanisms of structure-forming in dried drops of biological fluids [17,18], protein macromolecules retaining structure, physical, and chemical properties and conformation are concentrated in the concerned area of the sample. This allows to assume a decrease in the amount of native protein during intensive physical exercise, which corresponds to data from literature $[10,11]$. This is due to the fact that shifts in oxidative metabolism lead not only to the stimulation of lipid peroxidation in blood plasma and formed element membranes but also to oxidative modification of proteins $[8,10]$ resulting, in turn, in the loss of functionality, physical, and chemical properties of proteins.

In general, the study of crystallogenic properties of athlete blood serum along with the evaluation of oxidative metabolism modification in the biological fluid has verified negative shifts initiated by regular intensive physical exercise comparable in terms of direction with a blood biochemical response to this exposure.

This is also supported by the results of correlation analysis also confirming the presence of multiple relationships between the shifts in metabolic parameters representing the status of blood pro- and antioxidant systems and crystalloscopic parameters indicating changes in physical and chemical properties of biological fluids in professional athletes. These data additionally emphasize that an increase in the severity of oxidative stress (particularly manifested as an increase in 8-isoprostane level and concentrations of oLDLs and as a decrease in total antioxidant activity of plasma, activity of SOD and level of non-enzymatic antioxidants) takes place along with negative shifts in crystallogenic properties of the blood serum such as the increased activity of structure formation, increased degree of destruction in slide elements, and narrowed protein margin of the sample.

\section{CONCLUSION}

Therefore, the complex study demonstrated the presence of shifts in oxidative metabolism and blood serum physical and chemical properties induced by professional sports and manifested both in the status of blood pro- and antioxidant systems and biofluid crystallogenic activity. At the same time, the obtained data confirmed the development of oxidative stress in qualified athletes. However, irregularity of said metabolic transformations draws attention.

\section{AUTHORS' CONTRIBUTIONS}

Intellectual content, literature search, data analysis, manuscript preparation: Andrew K. Martusevich.

Data acquisition, literature search: Konstantin A. Karuzin.

\section{CONFLICTS OF INTEREST}

The authors declare that they have no conflicts of interest.

FUNDING

No funding was provided for this study.

\section{REFERENCES}

1. Åman M, Larsén K, Forssblad M, Näsmark A, Waldén M, Hägglund M. A nationwide follow-up survey on the effectiveness of an implemented neuromuscular training program to reduce acute knee injuries in soccer players. Orthop J Sports Med 2018;6:2325967118813841.

2. American College of Sports Medicine. American college of sports medicine position stand. Progression models in resistance training for healthy adults. Med Sci Sports Exerc 2009;41:687-708.

3. Tummala SV, Hartigan DE, Makovicka JL, Patel KA, Chhabra A. 10year epidemiology of ankle injuries in men's and women's collegiate basketball. Orthop J Sports Med 2018;6:2325967118805400.

4. Goodman AD, Etzel C, Raducha JE, Owens BD. Shoulder and elbow injuries in soccer goalkeepers versus field players in the national collegiate athletic association, 2009-2010 through 2013-2014. Phys Sportsmed 2018;46:304-11.

5. Reneker JC, Latham L, McGlawn R, Reneker MR. Effectiveness of kinesiology tape on sports performance abilities in athletes: A systematic review. Phys Ther Sport 2018;31:83-98.

6. Margonis K, Fatouros IG, Jamurtas AZ, Nikolaidis MG, Douroudos I, Chatzinikolaou A, et al. Oxidative stress biomarkers responses to physical overtraining: Implications for diagnosis. Free Radic Biol Med 2007;43:901-10.

7. Wagner KH, Reichhold S, Hölzl C, Knasmüller S, Nics L, Meisel M, et al. Well-trained, healthy triathletes experience no adverse health risks regarding oxidative stress and DNA damage by participating in an ultraendurance event. Toxicology 2010;278:211-6.

8. Halliwell BJ, Cutteridge MC. Free Radicals in Biology and Medicine. $3^{\text {rd }}$ ed. Oxford: Oxford University Press; 1999.

9. Veskoukis AS, Nikolaidis MG, Kyparos A, Kouretas D. Blood reflects tissue oxidative stress depending on biomarker and tissue studied. Free Radic Biol Med 2009;47:1371-4.

10. Aguiló A, Tauler P, Fuentespina E, Tur JA, Córdova A, Pons A. Antioxidant response to oxidative stress induced by exhaustive exercise. Physiol Behav 2005;84:1-7.

11. Ji L. Oxidative stress during exercise: Implication of antioxidant nutrients. Free Radic Biol Med 1995;18:1079-86.

12. Leelarungrayub D, Saidee K, Pothongsunun P, Pratanaphon S, Yankai A, Bloomer RJ. Six weeks of aerobic dance exercise improves blood oxidative stress status and increases interleukin-2 in previously sedentary women. J Bodyw Mov Ther 2011;15:355-62.

13. Morillas-Ruiz JM, García JA, López FJ, Vidal-Guevara ML, Zafrilla P. Effects of polyphenolic antioxidants on exercise-induced oxidative stress. Clin Nutr 2006;25:444-53.

14. Dreißigacker U, Wendt M, Wittke T, Tsikas D, Maassen N. Positive correlation between plasma nitrite and performance during high- 
intensive exercise but not oxidative stress in healthy men. Nitric Oxide 2010;23:128-35.

15. Statsenko EA. Characteristics of lipid peroxidation and markers of endogenous intoxication in monitoring physical loads during rower training. Vopr Kurortol Fizioter Lech Fiz Kult 2011;3:41-5.

16. Statsenko EA, Kovkova AV, Nekhaĭ EV. The development of a new marker of the training status in athletes. Vopr Kurortol Fizioter Lech Fiz
Kult 2012;3:42-5.

17. Martusevich AK, Kamakin NF. Crystallography of biological fluid as a method for evaluating its physicochemical characteristics. Bull Exp Biol Med 2007;143:385-8

18. Martusevich AK, Peretyagin SP. Modification of blood plasma crystallogenesis with nitrogen oxide processing. Biofizika 2013;58:1038-42. 